\title{
A Complex Case of Loeys-Dietz Syndrome: A Case Report and Review of the Literature
}

\author{
Tiffany N. Caza, Ana Mercedes, Robert Stoppacher, Charles A. Catanese
}

\begin{abstract}
Loeys-Dietz syndrome is a rare autosomal dominant connective tissue disorder with a genetic predisposition to aneurysm formation and congenital cardiofacial defects through genetic mutation affecting the transforming growth factor-beta (TGF $\beta$ ) signaling pathway. We present a case of a 6-year-old female patient with Loeys-Dietz syndrome who developed an annular aortic valve abscess and ascending aortic dissection due to Staphylococcus aureus endocarditis. Within this case, multiple complications from Loeys-Dietz syndrome were identified, including prosthetic valve infection, aneurysm formation and dissection, septic embolism, and aspiration pneumonia. The clinical presentation, pathologic findings, and pathophysiology will be discussed. Acad Forensic Pathol. 2016 6(4): 731-738
\end{abstract}

\section{AUTHORS}

Tiffany N. Caza MD PHD, State University of New York Upstate Medical University - Pathology

Roles: Project conception and/or design, data acquisition, analysis and/or interpretation, manuscript creation and/or revision, approved final version for publication, accountable for all aspects of the work.

Ana Mercedes, University of Nevada Las Vegas - Cardiology

Roles: Data acquisition, analysis and/or interpretation, manuscript creation and/or revision, approved final version for publication, accountable for all aspects of the work, writing assistance and/or technical editing.

Robert Stoppacher MD, Onondaga County Medical Examiner's Office

Roles: Project conception and/or design, data acquisition, analysis and/or interpretation, manuscript creation and/or revision, approved final version for publication, accountable for all aspects of the work, general supervision, writing assistance and/or technical editing.

Charles A. Catanese MD, Onondaga County Medical Examiners Office - Pathology

Roles: Project conception and/or design, data acquisition, analysis and/or interpretation, manuscript creation and/or revision, approved final version for publication, accountable for all aspects of the work, principal investigator of the current study, general supervision, writing assistance and/or technical editing.

\section{CORRESPONDENCE}

Tiffany N. Caza MD PHD, 750 East Adams Street, Department of Pathology, Syracuse NY 13210, cazat@upstate.edu

ETHICAL APPROVAL

As per Journal Policies, ethical approval was not required for this manuscript

STATEMENT OF HUMAN AND ANIMAL RIGHTS

This article does not contain any studies conducted with animals or on living human subjects

\section{STATEMENT OF INFORMED CONSENT}

No identifiable personal data were presented in this manuscsript

DISCLOSURES \& DECLARATION OF CONFLICTS OF INTEREST

This work was presented at the 2016 NAME Annual Meeting. The authors, reviewers, editors, and publication staff do not report any relevant conflicts of interest

FINANCIAL DISCLOSURE

The authors have indicated that they do not have financial relationships to disclose that are relevant to this manuscript

KEYWORDS

Forensic pathology, Loeys-Dietz syndrome, Annular abscess, Prosthetic valve endocarditis, Aortic dissection

INFORMATION

ACADEMIC FORENSIC PATHOLOGY: THE OFFICIAL PUBLICATION OF THE NATIONAL ASSOCIATION OF MEDICAL EXAMINERS

@2017 Academic Forensic Pathology International • (ISSN: 1925-3621) • https://doi.org/10.23907/2016.069

Submitted for consideration on 15 Oct 2016. Accepted for publication on 18 Nov 2016 


\section{INTRODUCTION}

Inherited connective tissue disorders, including Loeys-Dietz syndrome, Marfan syndrome, and Ehlers-Danlos syndrome, are characterized by genomic mutations and can lead to dilation and weakening of arterial walls, predisposing to aneurysmal rupture, dissection, and sudden cardiac death. In affected individuals, aortic surgery is often required to stabilize the aortic root to prevent rupture or dissection of aneurysms. A common route of aortic stabilization is via the Bentall procedure, where the aortic root is replaced with a porcine aortic valve attached to a Carpentier-Edwards conduit with a second conduit at the transverse arch anastomosed to the native transverse arch. While aortic root stabilization can prevent dissection, there is a risk of graft dehiscence in a growing child as well as a nidus for infection due to the presence of synthetic material.

History of previous cardiac valve surgery and a prosthesis are risk factors for development of infectious endocarditis (IE). Porcine prosthetic valves increase the risk of IE, aortic stenosis, and sudden death. Endocarditis of porcine valves usually affects the cusps, with annular infection being uncommon, but it can rarely occur. Damage to valvular endothelium results in platelet-fibrin deposition to heart valves, colonization, and formation of vegetations. Valves with vegetations have poor compliance, resulting in regurgitation of blood flow and increased pressure to the proximal vessel. The increased pressures within the aortic root cause further dilatation and increase the risk of dissection. Prosthetic valve infection, aortic root aneurysm and dissection, and septic emboli are all complications of inherited connective tissue disorders that can be fatal. Loeys-Dietz syndrome should be considered in the differential diagnosis, along with Marfan and vascular Ehlers-Danlos syndrome, when an aortic root aneurysm or dissection is seen in a child, adolescent, or young adult. Screening of family members should also be considered, due to the autosomal dominant inheritance pattern.

\section{CASE REPORT}

\section{Clinical Presentation}

The patient was a 6-year-old girl who had a clinical history of Loeys-Dietz syndrome, gastroesophageal reflux disease, and recurrent episodes of aspiration pneumonia. The diagnosis of Loeys-Dietz syndrome was confirmed by genetics and was consistent with Type I due to a transforming growth factor-beta (TGF $\beta$ ) receptor mutation. At the age of two years, she underwent aortic arch repair with porcine aortic valve replacement with a Bentall procedure. She also had undergone repair of a cleft palate through a multistaged operation. As a surgical complication, she developed unilateral complete vocal cord paralysis. Due to her history of cleft palate and vocal cord dysfunction, she had dysphagia and recurrent episodes of aspiration, resulting in malnutrition and failure to thrive.

She presented to the emergency department with fever and hypotension with suspected recurrent aspiration pneumonia. She was given $60 \mathrm{~mL} / \mathrm{kg}$ intravenous fluid boluses, stress steroids, and a dobutamine drip until normotensive with a systolic blood pressure in the 80s. Chest radiograph on admission showed cardiomegaly, aortic arch calcifications, bilateral pleural effusions with streaky infiltrates of the bilateral lung bases, and opacification of the right lung base, consistent with pneumonia. An echocardiogram showed aortic stenosis, which was unchanged from her baseline. She was empirically treated with piperacillin-tazobactam and vancomycin for pneumonia. Blood cultures grew methacillin-sensitive Staphylococcus aureus, and her antibiotics were switched to oxacillin, rifampin, and gentamicin. Prosthetic valve endocarditis was clinically suspected, given her increased risk and infection with $S$. aureus.

With intravenous antibiotic treatment, she became afebrile with negative blood cultures. However, pleural effusions on chest radiograph worsened and became dyspneic. She was given furosemide and digoxin. Over the course of admission, she had increased oxygen requirements to maintain oxygen saturation above $80 \%$. She had several episodes of desaturation 
below $80 \%$ and was intubated. She developed bradycardia and was given atropine without response. Resuscitation efforts began and several rounds of chest compressions with intermittent epinephrine dosing were provided. With continuation of chest compressions, bright red blood emerged from her endotracheal tube. Fresh frozen plasma, vitamin K, and activated factor VII (Novoseven) were given to control bleeding without response. The resuscitation efforts were ceased after one hour and she was declared dead. The family requested that an autopsy be performed.

\section{Autopsy Findings and Gross Pathology}

On external examination, the decedent appeared the stated age and had a thin body habitus with a body mass index (BMI) of $14 \mathrm{~kg} / \mathrm{m}^{2}$. Hypertelorism was noted. The teeth were overall in poor condition, with multiple capped teeth and absence of two upper and lower incisors. A white, healed scar was present along the upper lip consistent with a history of cleft lip and palate repair with no residual clefting present. The proximal interphalangeal joints of the bilateral hands had contractures at every digit. Clubfoot deformity was also noted. The neck was symmetrical, the trachea was midline, and the chest had a normal anteroposterior dimension with prominent pectus carinatum and a sternotomy scar at the site of prior cardiac surgery. Evidence of medical therapy was observed, including an endotracheal tube, peripherally inserted central catheter, hospital bracelets, and intravenous and intraosseous catheters.

On internal examination, all major organs were in their correct anatomic positions. The body cavities contained fibrous adhesions involving the pericardial sac (Image 1) and the right lung base. Bilateral serosangineous pleural effusions were present, measuring $260 \mathrm{~mL}$ on the right and $200 \mathrm{~mL}$ on the left. The heart and attached aorta weighed $250 \mathrm{~g}$. The heart showed levocardia with normal orientation of the great vessels. The coronary vasculature was right-dominant with no atherosclerosis noted. The right atrial chamber had moderate dilatation with moderately thickened trabeculae. A probe-patent foramen ovale was present with a $0.3 \mathrm{~cm}$ anterior-inferior defect. The tricuspid valve contained three leaflets, each with mild myxoid thickening, no vegetations, and measured $9.2 \mathrm{~cm}$ in circumference. The right ventricular myocardium was red-brown, $0.3 \mathrm{~cm}$ thick, and without dilatation. The pulmonic outflow tract was intact and the pulmonic valve contained three leaflets, measuring $6.4 \mathrm{~cm}$ in circumference. The ductus arteriosus was closed. The right and left pulmonary arteries coursed to the appropriate lungs and were each $0.7 \mathrm{~cm}$ in diameter. The right and left pulmonic veins connected to a normally developed left atrial chamber. The left atrium was not dilated and its mitral valve was intact without vegetations, measuring $9.5 \mathrm{~cm}$ in circumference. The aortic valve was replaced with a $16 \mathrm{~mm}$ bioprosthesis, and the leaflets contained granular, brown-red, friable material on all cusps. This was associated with a circumferential dissection surrounding the aortic valve that extended around a dilated $1.9 \mathrm{~cm}$ tube graft of ascending aorta with hemorrhage between the graft and surrounding connective tissue measuring 0.4 to $0.5 \mathrm{~cm}$ in thickness. The dissection extended to the native aorta and did not involve the coronary arteries or arch vessels. The great vessels were significant for a hypoplastic left subclavian artery that measured 0.2 $\mathrm{cm}$ in diameter.

The lungs were congested, with frothy fluid present within the tracheobronchial tree (Image 1). There was no connection present between the aortic graft and trachea; a fistula was not identified. The right lung contained four lobes and the left lung contained two lobes. The right and left lungs weighed 360 and $330 \mathrm{~g}$, respectively. Copious amounts of blood were extruded from the lung parenchyma on sectioning, and the lung parenchyma was red-purple. There were no focal lesions, areas of consolidation, or emphysematous changes present within the lungs. No saddle embolus was identified on examination of the pulmonary trunk. The pulmonary arteries were free from thromboemboli.

\section{Histopathology}

Sections of all major organs, including the heart, lungs, liver, stomach, small intestine, pancreas, spleen, kidney, lymph nodes, and bone marrow were examined by 
light microscopy. The right and left ventricular walls of the heart showed dilated blood vessels with mild chronic inflammation and granulation tissue at the epicardial surface. The cardiac muscle was thickened, with hypertrophied trabeculae carnae. Within the left ventricular myocardium, a septic embolus was identified, containing cellular debris, fibrin, and abundant acute inflammation. The mitral valve showed mild myxoid change with focal nodules of dense fibrotic collagen. The bioprosthetic aortic valve at the endocardial function showed a mixture of necrotic material, acute inflammation, bacterial colonies, and fibrin, consistent with an abscess (Image 2). A Grocott's methenamine silver stain was negative for yeast or fungi.
The acute inflammation extended into the valve tissue itself and partially involved adjacent fibrotic connective tissue with granulation tissue reaction. At the site of aortic dissection, there was accumulation of blood, neutrophils, and necrotic material on one side of the vessel lumen with graft material on the opposite side, consistent with dehiscence of the graft and ascending aortic dissection (Image 3).

Cross-sections of the aorta distal to the site of dissection, as well as the iliac arteries, showed no degeneration or aneurysm of the tunica media. No thickening of the tunica adventitia was identified. No atherosclerosis or vasculitis was noted.

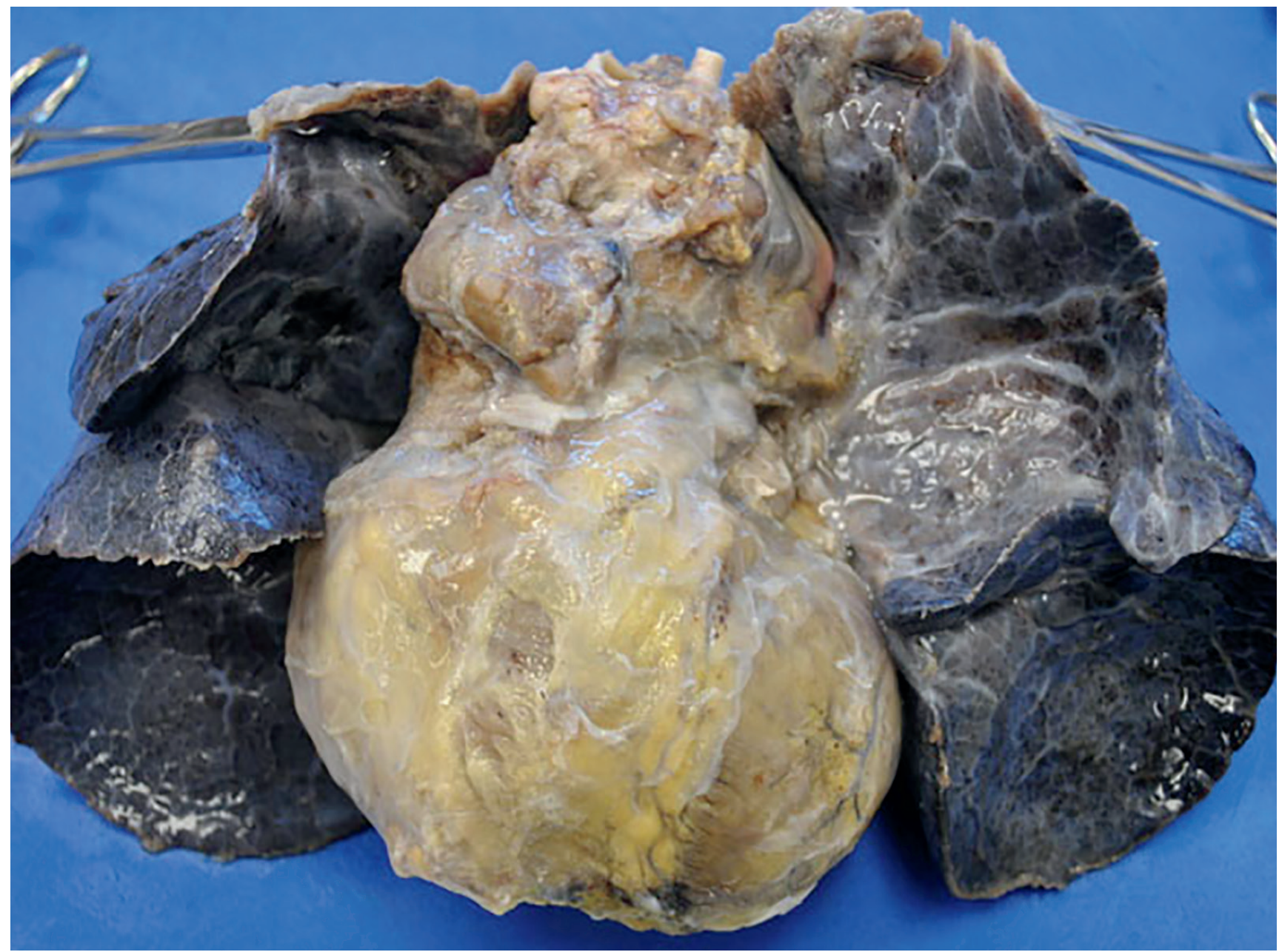

Image 1: Heart-lung block (post-fixation in 10\% formalin). Cardiomegaly was seen with thick, dense, fibrous pericardial adhesions. The lungs were diffusely congested. 

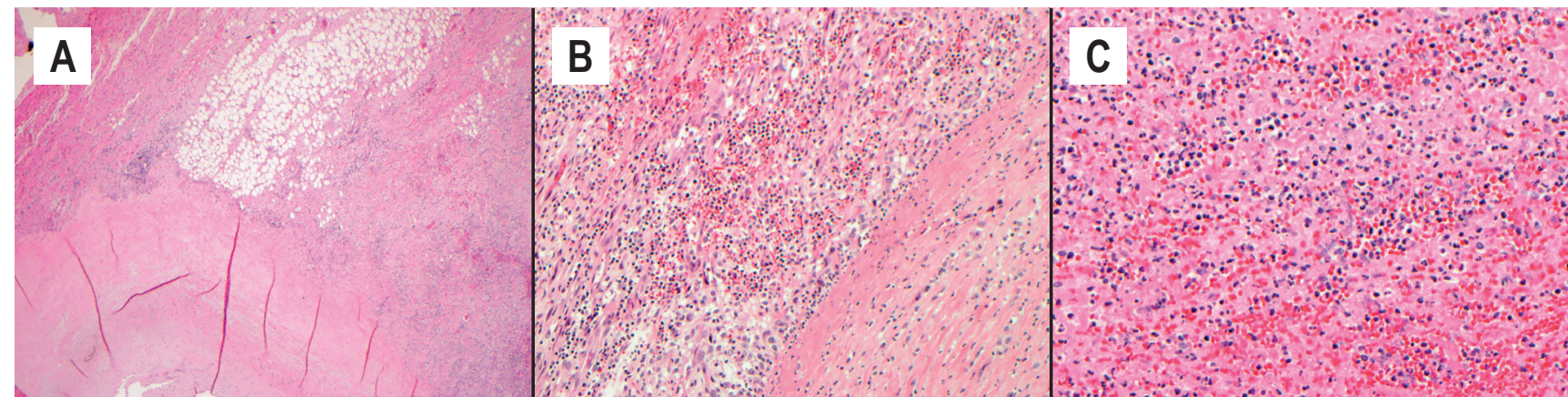

Image 2: A) The aortic valve at the endocardial junction shows presence of an abscess, with mixed acute and chronic inflammation and necrotic material (H\&E, x40). B) Surrounding the abscess is granulation tissue formation with an increase in fibroblasts, small blood vessel dilation, and an increase in vascular density. The acute inflammation extends to the endocardial surface (H\&E, $x 100)$. C) Abundant, friable material containing neutrophils, lymphocytes, macrophages, hemorrhage, and fibrin is present surrounding the aortic valve in a ring-like abscess (H\&E, x200).
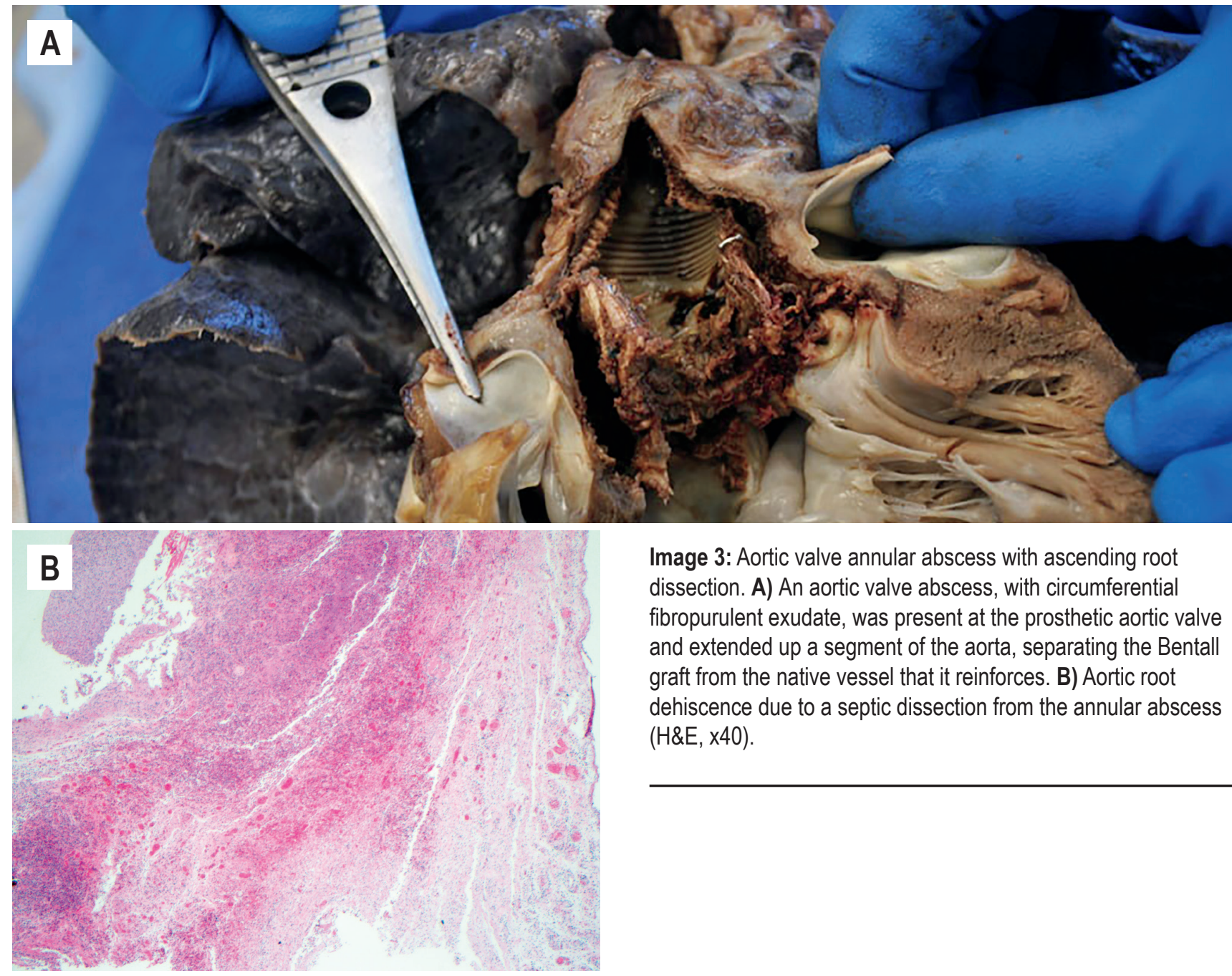

Image 3: Aortic valve annular abscess with ascending root dissection. A) An aortic valve abscess, with circumferential fibropurulent exudate, was present at the prosthetic aortic valve and extended up a segment of the aorta, separating the Bentall graft from the native vessel that it reinforces. B) Aortic root dehiscence due to a septic dissection from the annular abscess (H\&E, x40).

Page 735

Caza et al. - Complications of Loeys-Dietz Syndrome ACADEMIC FORENSIC PATHOLOGY: THE OFFICIAL PUBLICATION OF THE NATIONAL ASSOCIATION OF MEDICAL EXAMINERS (C2016 Academic Forensic Pathology International

Downloaded from www.afpjournal.com by an AFP Journal subscriber

This article is for personal use only and may not be shared or distributed in any fashion 
All lobes of the lungs showed peribronchial lymphoid aggregates, intraalveolar hemorrhage, and areas of atelectasis. The liver parenchyma had intact lobular architecture with prominent sinusoidal vessels. Mild lymphocytic perivascular cuffing was present. Centrilobular necrosis was present in association with mild acute and chronic inflammation near central veins. The stomach showed submucosal lymphoid aggregates and was without eosinophilia. The small intestine also showed prominent submucosal lymphoid follicles and patchy areas of hemorrhage. Granulation tissue, with abundant lymphocytes, plasma cells, and eosinophils, was present within the lamina propria. Eosinophilia did not exceed 15 cells/high power field. Sections of the pancreas and spleen were unremarkable. The kidneys showed proteinaceous material within dilated tubules, consistent with acute tubular necrosis. Rare sclerotic glomeruli were noted.

Lymph nodes showed lipogranulomatous change and contained a prominent reticular network. The germinal centers were reactive, appearing prominent and polarized. The bone marrow had appropriate cellularity for age (approximately 90\%), with intact trilineage hematopoiesis and a normal myeloid-to-erythroid ratio (3:1).

\section{Autopsy Diagnoses}

The cause of death was determined to be complications of Loeys-Dietz syndrome and the manner of death was determined to be natural. A schematic summary of the pathophysiology ofthe case is presented in Figure 1.

\section{DISCUSSION}

Loeys-Dietz syndrome is a rare autosomal dominant connective tissue disorder characterized by tortuous blood vessels with risk of aneurysm and dissection. It is a newly recognized disease, first described in 2005. Loeys-Dietz syndrome is caused by germline mutation in either TGFB2, TGFBR1, TGFBR2, or SMAD3 (1).

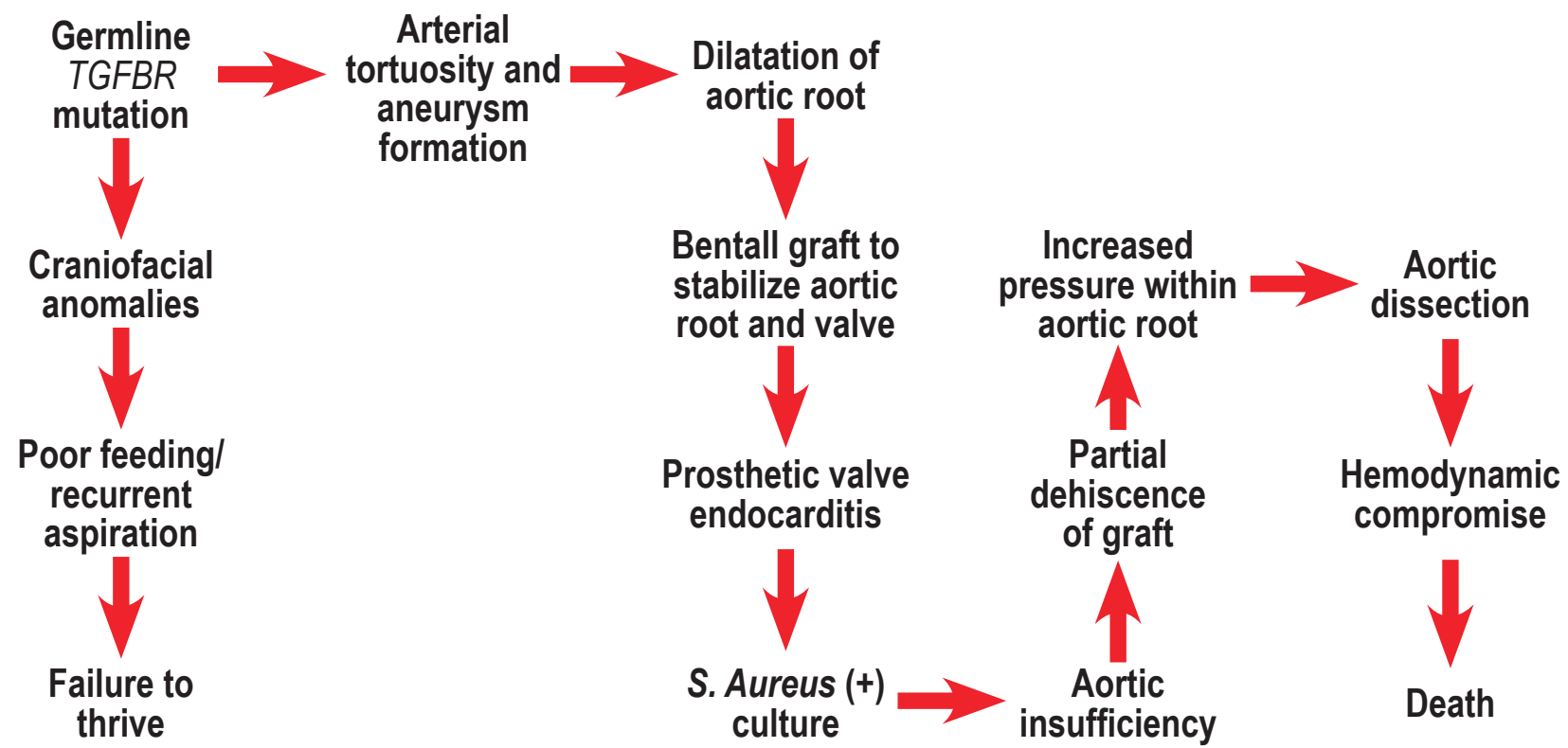

Figure 1: Pathophysiology of case. The patient's underlying TGF $\beta$ vasculopathy resulted in dilatation of the aortic root with aneurysm formation. Aortic root stabilization surgery with a Bentall graft was performed to strengthen the aortic root. Presence of a prosthetic valve increased the risk for colonization and infectious endocarditis. Staphylococcus aureus endocarditis resulted in aortic insufficiency and non-compliance of the aortic valve due to vegetations. The increased pressures in the aortic root and annular abscess of the aortic valve resulted in partial dehiscence of the graft. An aortic dissection resulted, leading to hemodynamic compromise, and death. 
Any of these mutations will result in aberrant TGF $\beta$ signaling (of the TGF $\beta$ related vasculopathies family), with an increase in active TGF $\beta$-mediated signal transduction. This is supported by increased TGF $\beta$ expression within diseased tissue from Loeys-Dietz syndrome patients (aortic and mitral valve) and by a reduction of aortic root growth in animal models when treated with anti-TGF $\beta$ neutralizing antibodies (2). This patient had a diagnosis of Loeys-Dietz syndrome confirmed by genetics and succumbed to complications of the disease.

The disease spectrum contains four subtypes that are based on the mutant gene that is present, although there is significant overlap in disease manifestations. In addition to aortic root dilatation, Type I Loeys-Dietz syndrome is categorized by craniofacial and skeletal abnormalities including hypertelorism, cleft palate, bifid uvula, thin body habitus, long extremities, and long slender fingers. These were all disease manifestations that we observed in this patient. Type I is the most serious form of the disease, with the highest cardiovascular risk and the lowest average lifespan (22 years) (3). Loeys-Dietz syndrome overlaps with other connective tissue disorders, in both the genes involved and the disease manifestations (mainly joint laxity and risk of aneurysm formation), including Marfan syndrome and Ehlers-Danlos syndrome (4), particularly in type II.

Numerous anatomic abnormalities are associated with Loeys-Dietz syndrome. Cardiovascular, craniofacial, skeletal, and cutaneous manifestations all can occur concomitantly. Cardiovascular manifestations include aneurysms and congenital heart defects. Aneurysms occur secondary to vascular tortuosity. The most common site of aneurysm is at the aortic root (present in $98 \%$ of patients), but can affect arterial branches within the head, neck, thoracic aorta, abdominal aorta, iliac arteries, and extremities as well (with greater than $50 \%$ having a dissection at a separate site). Congenital heart defects include a bicuspid aortic valve, atrial septal defect, patent ductus arteriosus, mitral valve insufficiency, mitral valve prolapse, and left ventricular hypertrophy (1). This patient suffered an aortic root aneurysm and dissection, consistent with the natural course of Loeys-Dietz syndrome.
The connective tissue defects lead to disease in numerous other organs in addition to the cardiovascular system in Loeys-Dietz syndrome. The skin has a thin, velvety, translucent appearance. Ophthalmologic abnormalities in Loeys-Dietz are common, with blue sclerae, cataracts, and a risk of retinal detachment. The eyes are often widely spaced, resulting in strabismus. Of note, the decedent in this case had a history of bilateral strabismus repair from her widely spaced eyes, cleft palate repair, finger contractures, pes planus, and clubfoot deformity - all features of Loeys-Dietz syndrome.

There are gastrointestinal abnormalities in Loeys-Dietz syndrome, with eosinophilic intestinal disease (esophagitis, gastritis, or colitis) leading to intestinal inflammation and food allergies. This can lead to failure to thrive and a need for nutritional supplementation to maintain body weight. There is a risk of prolapse of the bowel, uterus, and bladder in Loeys-Dietz syndrome, as well as a risk of intestinal rupture due to "hollow" organs. Additionally, cleft palate and vocal cord dysfunction can cause poor feeding with recurrent aspiration. The decedent had a history of recurrent episodes of aspiration pneumonia and ear infections, due to her history of cleft palate (status-post repair) and unilateral vocal cord paralysis. As a result, she had failure to thrive and a markedly low body weight $\left(\mathrm{BMI}=14 \mathrm{~kg} / \mathrm{m}^{2}\right)$. No eosinophilia was identified in microscopic examination of the stomach and small intestine, however.

Aortic surgery is often required early in life to prevent dissection of life-threatening aneurysms, with the timing and technique utilized dependent on the age of the patient, absolute dimension of the aorta, rate of progression of dilatation, genotype (greatest risk in Type I), and aortic valve function. In general, regardless of other factors, surgery is required if the aortic root is greater than or equal to $4 \mathrm{~cm}$ in diameter. Aortic valve replacement is often done with porcine valves to avoid requiring lifelong anticoagulation in pediatric patients. In addition to surgical repair, life-long treatment of angiotensin receptor blockers (ARBs) is indicated to reduce hemodynamic stress on the blood vessels and to abrogate TGF $\beta$ signaling. The patient 
was taking an ARB and was status-post aortic root repair by a Bentall procedure. In this procedure, the aortic root is replaced with a $16 \mathrm{~mm}$ porcine aortic valve attached to a Carpentier-Edwards conduit with a second conduit at the transverse arch anastomosed to the native transverse arch.

Presence of the graft material increases the risk of infection and development of endocarditis. The decedent had a porcine aortic valve placed due to severe aortic stenosis and insufficiency, which later became infected with Staphylococcus aureus. S. aureus infection is not an uncommon causative agent in bioprosthetic valve endocarditis, being identified in $10 \%$ of all infections of bioprostethic valves (5). Endocarditis of porcine valves usually affects the cusps, with annular infection being uncommon, although it has been reported (6). The cusps contained vegetations, as well as an annular ring abscess affecting the entire valve. Therefore, it was a combination of endocarditis and aortic dissection that resulted in death. Aortic valve endocarditis led to aortic insufficiency and stenosis with partial dehiscence of the graft material. The increased pressures within the aortic root caused further dilatation, surrounding the graft, leading to dissection of the aorta, hemodynamic decompensation, and death. The aortic dissection did not involve a coronary or carotid artery, and the amount of hemorrhage at the site of dissection alone would not cause hemodynamically significant hypovolemia. Acute on chronic aortic insufficiency was the mechanism of death, leading to the heart acting as an ineffective pump. The chronic aortic insufficiency was due to chronic aortic root dilatation. The acute aortic insufficiency was due to fibrinoid necrosis of the valve from infectious endocarditis. In a patient with low reserve, we believe that the aortic dissection could have led to the patient's death, as the patient already suffered from severe aortic insufficiency.

\section{CONCLUSION}

If Loeys-Dietz syndrome is suspected in a previously undiagnosed decedent, genetic testing should be considered. Genetic testing for Loeys-Dietz syndrome involves a core four gene sequencing panel, including the genes $S M A D 3, T G F B 2, T G F B R 1$, and TGFBR2 which cover mutations present in each of the four subtypes of Loeys-Dietz syndrome. Some tests also add FBN1 mutated in Marfan syndrome and/or COL3Al mutated in Ehlers-Danlos syndrome. Three to five mililiters of fresh whole blood with EDTA anticoagulant (K2EDTA or K3EDTA tubes) is required for the analysis. Hemolyzed and clotted specimens are unable to be used; however, postmortem samples can be tested if the DNA quality is adequate. A skin biopsy can be used in place of whole blood as the source of DNA for the testing in decedents who had recent transfusion or do not have nonhemolyzed blood available for analysis. Several laboratories will perform testing on fresh samples to assess family members, which is recommended once confirmed, as Loeys-Dietz syndrome has autosomal dominant inheritance.

\section{REFERENCES}

1) MacCarrick G, Black JH 3rd, Bowdin S, et al. Loeys-Dietz syndrome: a primer for diagnosis and management. Genet Med. 2014 Aug; 16(8):576-87. PMID: 24577266. PMCID: PMC4131122. https://dx.doi.org/10.1038/gim.2014.11

2) De Backer J, Campens L, De Paepe A. Genes in thoracic aortic aneurysms/dissections - do they matter? Ann Cardiothorac Surg. 2013 Jan; 2(1):73-82. PMID: 23977562. PMCID: PMC3741818. https://dx.doi.org/10.3978/j.issn.2225-319X.2012.12.01.

3) Loeys BL, Dietz HC. The Loeys-Dietz syndrome. Arch Dis Child. 2007 Feb; 92 (2):119. https://doi.org/10.1136/adc.2006.110593.

4) Aalberts JJ, van den Berg MP, Bergman JE, et al. The many faces of aggressive aortic pathology: Loey-Dietz syndrome. Neth Heart J. 2008 Sep; 16(9):299-304. PMID: 18827873. PMCID: PMC2553155. https://doi.org/10.1007/bf03086168.

5) Ivert TS, Dismukes WE, Cobbs CG, et al. Prosthetic valve endocarditis. Circulation. 1984 Feb; 69(2):223-32. PMID: 6690095.

6) Girgis RE, Rosman H, del Busto R, et al. Porcine bioprosthetic aortic valve endocarditis with ring abscess and aortic stenosis. Henry Ford Hosp Med J. 1991; 39(2):123-5. PMID: 1890006. 